\title{
Coupling of ion and network dynamics in lithium silicate glasses: a computer study
}

\author{
Magnus Kunow* and Andreas Heuent \\ Institut für Physikalische Chemie and Sonderforschungsbereich 458, \\ Westfälische Wilhelms-Universität, Corrensstraße 30, D-48149 Münster, Germany
}

(Dated: November 20, 2018)

\begin{abstract}
We present a detailed analysis of the ion hopping dynamics and the related nearby oxygen dynamics in a lithium meta silicate glass via molecular dynamics simulation. For this purpose we have developed numerical techniques to identify ion hops and to sample and average dynamic information of the particles involved. This leads to an instructive insight into the microscopic interplay of ions and network. It turns out that the cooperative dynamics of lithium and oxygen can be characterized as a sliding door mechanism. It is rationalized why the local network fluctuations are of utmost importance for the lithium dynamics.
\end{abstract}

*Electronic address: kunow@uni-muenster.de

${ }^{\dagger}$ Electronic address: andheuer@uni-muenster.de 


\section{INTRODUCTION}

As a well known characteristic feature of ion conducting glasses the dynamics of ions and network atoms are strongly decoupled at low temperatures and in particular below the glass transition temperature $T_{\mathrm{g}}[1,2,3]$. Although by definition for temperatures $T<T_{\mathrm{g}}$ the network dynamics is mainly limited to localized fluctuations, molecular dynamics simulations of alkali silicate systems with an immobilized network give clear evidence that the network dynamics has a significant impact on the ion dynamics [4, 5, 6, 7]. A similar behavior has already been predicted by Anderson and Stuart who have assumed that the opening of diffusion pathways between adjacent ionic sites through the silicate matrix supplies a major contribution to the activation energy of the ion diffusion in alkali silicate glasses [8, 9]. In recent work it has been proposed that at least for mixed-alkali systems a strong coupling of alkali and network dynamics should be present to rationalize experimental findings [10]. Furthermore for models of ion dynamics it is particularly important to know the nature of the network relaxation if an ion is leaving its site [11].

In 7] we have recently shown that for a lithium silicate system with an artificially immobilized silicate network the lithium dynamics changes in many aspects compared to a system with unchanged network mobility. A dramatic slowing down of the ion dynamics is observed, leading to an increase of the activation energy for the lithium diffusion by a factor of 1.7. Furthermore, it has turned out to be the immobilization of the oxygen particles that contributes most to this behavior. Analysis of suitable three-time-correlation functions has shown that forward-backward correlations of the lithium dynamics is much more pronounced. Thus, the minima of the potential landscape inside the fixed network structure act on the ions like an effective harmonic potential for significantly larger displacements of the ions than this would be the case inside a network carrying out its natural dynamics. Furthermore, by immobilizing the network species the lithium dynamics gets more heterogeneous. Recently, also the effect of immobilization of a few ions on the the dynamics of the remaining ions has been studied [12].

Another important ingredient for the ion dynamics through the glass matrix concerns the

availability of a vacant site in the neighbourhood of an ion. This problem gains particular importance if the number density of ions is close the number density of sites. From molecular dynamics simulations by Lammert et al., Habasaki et al. and Vogel it has turned out that 
in the lithium silicate system investigated in this work the number density of sites is only few percent larger than the one of ions [6, 13, 14]. This result is consistent with the previous observation of ion channels [15]. Thus, for most ions there is no adjacent vacant site to which it might hop. These ions have to wait for an adjacent ion to perform a hop leaving a vacant side before they can perform a hop on their own. Thus, a macroscopic charge transport requires some cooperative hopping mechanism. Such correlated subsequent hops have been observed by Cormack et al. simulating a sodium silicate system [16]. For lithium silicate correlated subsequent hops have been found by Habasaki et al., especially for ions with high mobility [17, 18, 19, 20]. They traced this behavior back to the prevention of a correlated backward hop due to the re-occupation of the initial site.

The goal of this work is to identify ion hops and sample the relevant dynamic information. Our analysis goes beyond the observation of individual hopping scenarios. By calculating averages over many different hopping events (of the order $10^{5}$ ) we are able to make statistically relevant statements about the nature of the dynamics.

We have organized this paper as follows. In sec. II we describe the technical aspects of the simulation and present the details of our analysis. The results are shown in sec. III. Finally, conclusions are drawn and discussed in sec. IV.

\section{TECHNICAL ASPECTS}

\section{A. Simulation}

The potential energy of the lithium silicate system is chosen to be the sum of a Buckingham and a Coulomb pair potential:

$$
U_{i j}\left(r_{i j}\right)=\frac{1}{4 \pi \epsilon_{0}} \frac{q_{i} q_{j}}{r_{i j}}-\frac{C_{i j}}{r_{i j}^{6}}+A_{i j} \exp \left(-B_{i j} r_{i j}\right)
$$

The indices $i$ and $j$ denote the species lithium, oxygen, and silicon, respectively. The potential parameters are listed in previous work by Banhatti and Heuer [21]. They are based on ab initio calculations by Habasaki et al. [22, 23, 24]. Applying a slightly modified version 2.16e of the MOLDY software package [25], we have generated molecular dynamics (MD) trajectories in the NVT ensemble, i.e. at constant volume. The length of the elementary time step has been chosen to be 2 fs and periodic boundary conditions have been used. We 
have simulated a system of $N=1152$ particles at a density $\rho=2.34 \mathrm{~g} \mathrm{~cm}^{-3}$ taken from experimental room temperature data [26]. If the temperature is not explicitly noted the presented data refers to $T=980 \mathrm{~K}$.

We have also modified the MOLDY software to compute only the dynamics of a chosen subensemble of particles and keep the positions of the other particles constant. Doing so, we are able to take a configuration of our system at an arbitrarily chosen time and continue the dynamics only for the ions so that the network is artificially immobilized.

\section{B. Definition of lithium hops}

Qualitatively, a hop is identified as a relatively large displacement during a relatively short time. Thus, we take a minimum displacement $s_{\min }$ occurring during a time interval $2 \tau$ as the criterion for an ion hop. As we are interested in hops between next neighbor positions the value of $s_{\min }$ ought to be somewhat larger than the first minimum distance $r_{\min } \approx 1.5 \AA$ of the self part of the van Hove correlation function and somewhat less than the average hopping distance $d_{0} \approx 2.6 \AA[3]$. The average time an ion resides outside a lithium site has been found to be 500 fs [13]. The total time of the hop, also including the time to leave the initial site and to enter its new site is somewhat larger. Here we choose $\tau=1$ ps. The actual choice is uncritical (see below).

Let us assume $t_{0}$ to be the time when a hopping ion is crossing the saddle point between two adjacent sites. To identify the position $\mathbf{r}_{\mathrm{sl}}$ of this saddle as well as the position $\mathbf{r}_{\text {init }}$ of the initial lithium site and $\mathbf{r}_{\text {end }}$ of the lithium site after the hop, it has turned out to be very useful to average the position of the ion at times $t_{0}, t_{0}-\tau, t_{0}+\tau$, respectively, over a short time. Since a priori $t_{0}$ is not known we define for these averages:

$$
\begin{aligned}
\mathbf{r}_{\mathrm{sl}}(t) & =\frac{1}{2 n_{\mathrm{sl}}+1} \sum_{i=-n_{\mathrm{sl}}}^{n_{\mathrm{sl}}} \mathbf{r}\left(t+i t_{\text {slice }}\right) \\
\mathbf{r}_{\text {init }}(t) & =\frac{1}{2 n_{\text {init }}+1} \sum_{i=-n_{\text {init }}}^{n_{\text {init }}} \mathbf{r}\left(t-\tau+i t_{\text {slice }}\right), \\
\mathbf{r}_{\text {end }}(t) & =\frac{1}{2 n_{\text {end }}+1} \sum_{i=-n_{\text {end }}}^{n_{\text {end }}} \mathbf{r}\left(t+\tau+i t_{\text {slice }}\right)
\end{aligned}
$$

$t_{\text {slice }}=20 \mathrm{fs}$ is the time between subsequent system configurations, stored during the MD run. Using $n_{\mathrm{sl}}=5$ and $n_{\text {init }}=n_{\text {end }}=25$ the position of the saddle point is averaged over 
$200 \mathrm{fs}$, and the positions of the lithium sites are averaged over 1 ps.

How to find an appropriate choice for $t_{0}$ ? For this purpose we evaluate eqs. (2.2) to (2.4) for all available times $t$ and for every lithium ion. For an ion hop one expects:

$$
\left|\mathbf{r}_{\text {end }}-\mathbf{r}_{\text {init }}\right| \geq s_{\text {min }}
$$

For a single hopping event one typically finds a set of subsequent points in time $t$ which do all fulfil the condition (2.5). We identify the exact time $t_{0}$ of the hop as the time $t$ when the hopping lithium ion is half-way between the initial site and the new site. Thus, the best value $t_{0}$ representing this particular ion hop is chosen by the criterion:

$$
|| \mathbf{r}_{\mathrm{end}}(t)-\mathbf{r}_{\mathrm{sl}}(t)|-| \mathbf{r}_{\mathrm{sl}}(t)-\mathbf{r}_{\mathrm{init}}(t) \|=\min
$$

The average hopping distance $\left\langle\left|\mathbf{r}_{\text {end }}-\mathbf{r}_{\mathrm{sl}}\right|\right\rangle$ is closest to $d_{0}$ with the choice $s_{\min }=2 \AA$. Why is $\tau=1$ ps a good choice? An appropriate criterion for the choice of $\tau$ is that the number of identified lithium hops is a maximum. It turns out that the number of identified jumps is basically constant for $0.7 \mathrm{ps} \leq \tau \leq 1.3$ ps. Analyzing a simulation run of $20 \mathrm{~ns}$ length at $T=980 \mathrm{~K}$ with this method we find more than 130000 ionic hops.

\section{Coordinate Frame}

We wish to study the densities of the ions and the network species (see section ID) in the temporal and spatial surrounding of each identified lithium hop. To average over all hops we need to define frames of reference that provide a certain sensitivity to the characteristic changes we are interested in. First, we define a time scale relative to $t_{0}$ :

$$
t_{\mathrm{S}}=t-t_{0}
$$

As we are interested in dynamic events near the saddle point and the initial site we introduce difference vectors relative to the position of the saddle point and the initial site, respectively, i.e.

$$
\mathbf{r}_{\mathrm{S}}=\mathbf{r}-\mathbf{r}_{\mathrm{sl}}
$$

and

$$
\mathbf{r}_{\mathrm{I}}=\mathbf{r}-\mathbf{r}_{\text {init }} .
$$


Obviously, one could also define a position vector $\mathbf{r}_{\mathrm{E}}$ relative to the position of the ion site after the hop, but due to time reversal symmetry, this would not lead to any new information.

It seems to be most instructive to describe the position vectors $\mathbf{r}_{\mathrm{S}}$ and $\mathbf{r}_{\mathrm{I}}$ in polar coordinates:

$$
\mathbf{r}_{\mathrm{S}}=\left(\begin{array}{c}
r_{\mathrm{S}} \\
\theta_{\mathrm{S}} \\
\phi_{\mathrm{S}}
\end{array}\right), \quad \mathbf{r}_{\mathrm{I}}=\left(\begin{array}{c}
r_{\mathrm{I}} \\
\theta_{\mathrm{I}} \\
\phi_{\mathrm{I}}
\end{array}\right)
$$

Here, the angle between the hopping vector $\mathbf{r}_{\mathrm{end}}-\mathbf{r}_{\text {init }}$ and the particular position vector is denoted by $\theta_{\mathrm{S}}$ and $\theta_{\mathrm{I}}$, respectively. $\phi_{\mathrm{S}}$ and $\phi_{\mathrm{I}}$ describe the angle in the plane perpendicular to the hopping vector. For reasons of isotropy no dependence on $\phi_{\mathrm{S}}$ or $\phi_{\mathrm{I}}$ is expected. The definitions are visualized in fig. 1.

\section{Definition of the investigated density functions}

Let $\left\langle\rho_{i}\left(r_{\mathrm{S}}, \theta_{\mathrm{S}}, t_{\mathrm{S}}\right)\right\rangle$ denote the number density of particles at time $t_{\mathrm{S}}$ belonging to species $i$ inside a small volume element characterized by $r_{\mathrm{S}}$ and $\theta_{\mathrm{S}}$ and averaged over all identified lithium hops. This quantity has been investigated for the hopping lithium ion (HLi) and the non-bridging oxygens (NBO). Actually, we also analyzed the bridging oxygens and the adjacent lithium ions, but these results will not be shown explicitly in this paper. By relating the values of $\left\langle\rho_{i}\left(r_{\mathrm{S}}, \theta_{\mathrm{S}}, t_{\mathrm{S}}\right)\right\rangle$ to the total number density $\bar{\rho}_{\mathrm{Li}}$ of lithium or $\bar{\rho}_{\mathrm{O}}$ of oxygen particles, respectively, one ends up with:

$$
\begin{gathered}
\mathrm{P}_{\mathrm{HLi}}\left(r_{\mathrm{S}}, \theta_{\mathrm{S}}, t_{\mathrm{S}}\right)=\left\langle\rho_{\mathrm{HLi}}\left(r_{\mathrm{S}}, \theta_{\mathrm{S}}, t_{\mathrm{S}}\right)\right\rangle / \bar{\rho}_{\mathrm{Li}} \\
\mathrm{P}_{\mathrm{NBO}}\left(r_{\mathrm{S}}, \theta_{\mathrm{S}}, t_{\mathrm{S}}\right)=\left\langle\rho_{\mathrm{NBO}}\left(r_{\mathrm{S}}, \theta_{\mathrm{S}}, t_{\mathrm{S}}\right)\right\rangle / \bar{\rho}_{\mathrm{O}} .
\end{gathered}
$$

\section{RESULTS}

\section{A. Lithium dynamics}

The quantity $\mathrm{P}_{\mathrm{HLi}}\left(r_{\mathrm{S}}, \theta_{\mathrm{S}}, t_{\mathrm{S}}\right)$ is plotted in fig. 2 for five different times $-2 \mathrm{ps} \leq t_{\mathrm{S}} \leq 0$. By definition the contributing ions perform a hop from the left to the right. The time evolution is as follows. 1.) At $t_{\mathrm{S}}=-2 \mathrm{ps} \mathrm{P}_{\mathrm{HLi}}$ shows a pronounced maximum at the initial site around $r_{\mathrm{S}} \approx 1.2 \AA$ and $\theta_{\mathrm{S}}=\pi$. From there an area of high density of hopping lithium spans the 
saddle along the axis $\theta_{\mathrm{S}}=0$ and reaches into the new site up to $r_{\mathrm{S}} \approx 1 \AA$. This reflects the presence of lithium ions which are about to hop from the site at $\mathbf{r}_{\text {end }}$ to $\mathbf{r}_{\text {init }}$ and, of course, will jump back at $t_{\mathrm{S}}=0$. Thus this intensity reflects back-and-forth dynamics. 2.) At $t_{\mathrm{S}}=-500 \mathrm{fs}$ the area where the density of hopping lithium is finite cumulates around the initial site. The maximum of $\mathrm{P}_{\mathrm{HLi}}$ has moved into the direction of the saddle and is now located at $r_{\mathrm{S}} \approx 1 \AA$ and $\theta_{\mathrm{S}}=\pi$. 3.) At $t_{\mathrm{S}}=-120$ fs $\mathrm{P}_{\mathrm{HLi}}$ shows a broad maximum along the direction of the hopping vector between the initial site and the saddle point with center at $r_{\mathrm{S}} \approx 0.7 \AA$ and $\theta_{\mathrm{S}}=\pi$. The density is strongly concentrated around this maximum. 4.) At $t_{\mathrm{S}}=-40$ fs the maximum concentration is close to $r_{\mathrm{S}} \approx 0.4 \AA$ and $\theta_{\mathrm{S}}=\pi$. 5.) Finally, at $t_{\mathrm{S}}=0$ the density is radially distributed around the saddle point. It is maximal within a radius $r_{\mathrm{S}} \approx 0.3 \AA$ and decays rapidly for larger values of $r_{\mathrm{S}}$. Due to the fact that the definition of $\mathbf{r}_{\mathrm{sl}}$ involves an average over several time steps, finite values of $r_{S}$ are possible for $t_{0}=0$.

Of course, the cumulation of the density of hopping lithium while $t_{\mathrm{S}}$ approaches zero is due to the fact, that by definition an ion is crossing the saddle close to $t_{\mathrm{S}}=0$. The broadening at earlier times shows that different ions behave quite differently in the time interval just before the hop.

Emphasizing the effect of highly occupied lithium sites, it has turned out that at least for a short time during the shown time interval $-2 \mathrm{ps} \leq t_{\mathrm{S}} \leq 0$ the site close to $\mathbf{r}_{\mathrm{end}}$ is occupied by another ion for about $83 \%$ of all identified lithium hops. This does not only imply a high probability to find an ion at a site almost immediately before another ion will perform a hop to this site. It also means a high probability for a site to be occupied again by an ion just after another ion has hopped out of it. We found the time scale of this re-occupation to increase slightly with decreasing temperature, but even at the significantly lower temperature $T=640 \mathrm{~K}$ more then $65 \%$ of the initial sites have been re-occupied by another ion at $t_{\mathrm{S}}=2 \mathrm{ps}$ (not shown).

\section{B. Oxygen dynamics around the saddle}

Before we focus on the oxygen dynamics during an ion hop, we show the partial pair correlation functions $g_{\mathrm{LiNBO}}(r)$ for lithium and non-bridging oxygen (fig. 31). $g_{\mathrm{LiNBO}}(r)$ is

different from zero for distances $r \gtrsim 1.6 \AA$. A pronounced maximum is found at $r \approx 2.0 \AA$ 
representing the first shell of non-bridging oxygen around lithium. The maxima for the second and higher order shells of non-bridging oxygen are less pronounced.

We can now compare this distance with characteristic distances $r_{\mathrm{S}}$ of $\mathrm{P}_{\mathrm{NBO}}\left(r_{\mathrm{S}}, \theta_{\mathrm{S}}, t_{\mathrm{S}}\right)$. The time evolution of $\mathrm{P}_{\mathrm{NBO}}$ is shown in fig. 4. 1.) At $t_{\mathrm{S}}=-2$ ps the density of nonbridging oxygen indicates two minima around the centers of the two lithium sites. The minimum around the initial site seems to be a little more pronounced. This matches the slightly but clearly higher lithium density at this site. $\mathrm{P}_{\mathrm{NBO}}$ shows a broad maximum around $r_{\mathrm{S}} \approx 1.8 \AA$ and $\theta_{\mathrm{S}} \approx \pi / 2$. The shortest distance to the saddle where $\mathrm{P}_{\mathrm{NBO}}$ reaches zero is at $r_{\mathrm{S}} \approx 0.6 \AA$. 2.) While time approaches zero the minima in non-bridging oxygen density get more and more pronounced. At the same time the density spike for $\theta_{\mathrm{S}} \approx \pi / 2$ separating the two minima vanishes. The maximum of $\mathrm{P}_{\mathrm{NBO}}$ broadens in $\theta_{\mathrm{S}}$ direction and narrows in $r_{\mathrm{S}}$ direction. Its peak increases and moves to slightly higher values of $r_{\mathrm{S}}$. At $t_{\mathrm{S}}=0$ nearly everywhere inside an approximately spherical volume with radius $r_{\mathrm{S}} \approx 1.5 \AA$ around the saddle the density of non-bridging oxygen has vanished. The maximum is shifted to $r_{\mathrm{S}} \approx 1.9 \AA$. A similar behavior is observed for the bridging oxygens.

The time evolution of $\mathrm{P}_{\mathrm{NBO}}\left(r_{\mathrm{S}}, \theta_{\mathrm{S}}, t_{\mathrm{S}}\right)$ gives evidence that during an ion hop the oxygen particles around the saddle point significantly retreat. This mechanism can be compared to a sliding door which has to be opened to let the ion pass. To investigate this effect in more detail, we show another plot of $\mathrm{P}_{\mathrm{NBO}}$, in which we concentrate on a thin slice of $0.5 \AA$ thickness perpendicular to the hopping vector around $r_{\mathrm{S}}=0$; see again fig. 1] The results are shown in fig. 5. We have also included the corresponding data for a system with an immobilized network at the same temperature $T=980 \mathrm{~K}$.

As the most pronounced effect oxygen density for $r_{\mathrm{S}}<1.5 \AA$ is dramatically reduced when $t_{\mathrm{S}}$ approaches zero. In case of the immobilized network the density of NBOs is also negligible for $r_{\mathrm{S}}<1.5 \AA$. Fig. 5 again shows the sliding door mechanism as already mentioned in the discussion of fig. 4. It seems to be related to a critical minimum distance between oxygen and the saddle at the time it is crossed by the hopping ion. This is $r_{\mathrm{S}} \approx 1.5 \AA$ for nonbridging oxygen. Very close to this value the respective pair correlation functions decays to zero (see fig. 3). The remaining small differences do not necessarily mean that during a hop the distance between lithium and non-bridging oxygen falls below $1.6 \AA$, because the ion does not have to permeate the plane around the saddle strictly at $\mathbf{r}_{\mathrm{S}}=0$.

To investigate the sliding door mechanism somewhat closer we analyze the distribution of 
non-briding oxygen atoms at $t_{\mathrm{S}}=0$ for different subensembles. Subensemble $A_{1}$ is defined as the set of oxygens with distances $r_{\mathrm{S}} \leq 1.5 \AA$ from a saddle at time $t_{\mathrm{S}}=-1$ ps. Subensemble $A_{2}$ is defined via the same criterion, but at time $t_{\mathrm{S}}=-2$ ps. The oxygen-distribution of

subensemble $A_{2}$ at time $t_{\mathrm{S}}=-1 \mathrm{ps}$ is distributed around $r_{\mathrm{s}} \approx 1.5 \AA$ and is therefore very different to subensemble $A_{1}$ at that time $\left(r_{\mathrm{S}} \leq 1.5 \AA\right)$; see fig. 6. This difference reflects the typical fluctuations of the oxygen atoms during 1 ps without an ion hop. Now the interesting question emerges whether the oxygen distribution at $t_{\mathrm{S}}=0 \mathrm{ps}$ is different for both subensembles. As also shown in fig. 6] both distributions are identical. Comparison with $\mathrm{P}_{\mathrm{NBO}}\left(r_{\mathrm{S}}, t_{\mathrm{S}}\right)$ for all non-bridging oxygens at time $t_{\mathrm{S}}=0$ shows that this distribution is very similar to the overall distribution at $t_{\mathrm{S}}=0$ after appropriate scaling to the number of contributing particles. Thus on average the position of oxygens close to the saddle at time $t_{\mathrm{S}}=0$, i.e. the time of the lithium hop, does not depend on its position in the past. Furthermore it turns out that the oxygen distribution $\mathrm{P}_{\mathrm{NBO}}\left(r_{\mathrm{S}}, t_{\mathrm{S}}=0\right)$ only weakly depends on temperature, as shown from the comparison of $T=980 \mathrm{~K} \mathrm{~K}$ with $T=640 \mathrm{~K}$. The implications of these observations will be discussed below.

\section{Oxygen dynamics around the initial site}

In fig. 7 we show the time evolution of the relative density $\mathrm{P}_{\mathrm{NBO}}\left(r_{\mathrm{I}}, t_{\mathrm{S}}\right)$ of non-bridging oxygen inside a hemisphere around $\mathbf{r}_{\text {init }}$ covering angles $\pi / 2 \leq \theta_{\mathrm{I}} \leq \pi$. At time $t_{\mathrm{S}}=-1$ ps the first maximum for $\mathrm{P}_{\mathrm{NBO}}\left(r_{\mathrm{I}}, t_{\mathrm{S}}\right)$ is found at $r_{\mathrm{I}} \approx 2.1 \AA$. The density decays to zero at $r_{\mathrm{I}} \approx 1.4 \AA$. When time $t_{\mathrm{s}}$ approaches zero, i.e. the ion leaves the initial site, the corresponding peak gets broader and slightly shifts to larger distances. This trend stops around $t_{\mathrm{S}} \approx 120 \mathrm{fs}$. For larger times the density tends to approach the initial density before the jump. The reason is that very likely the initial site will be populated by another ion. In summary, two major effects result after vacating an ion site. First, the network structures somewhat loosens as reflected by the broadening of the distribution and, second, the surrounding oxygen atoms slightly increase their distance from this site. 


\section{CONCLUSIONS}

In this paper we have introduced a useful tool to identify the time and the position of ion hops. This enables us to study the temporal and spatial evolution of the relative density distribution of hopping ions and adjacent oxygen atoms. This leads to an instructive picture of the microscopic ion hopping dynamics. It turns out that there is a high probability for a site to be re-occupied by an ion almost immediately after it has been left. Even for the lowest investigated temperature $T=640 \mathrm{~K}$ this re-occupation takes place on a time scale of picoseconds. This behavior is similar to results obtained for a sodium silicate system by Cormack et al. [16]. It also matches the perception of Habasaki et al. [17, 18, 19, 20].

We have shown that oxygen atoms near the saddle point of an ion hop retreat when the hop occurs. The oxygen motion reminds of a sliding door that opens to let the ion pass. Here a minimum distance to the saddle is kept by the oxygen which is very close to the distance where the corresponding pair correlation function with lithium vanishes.

This cooperative dynamics between lithium and those oxygen atoms close to the saddle can be easily represented in an effective 2D-energy landscape of the type as sketched in fig. 8. Of course, in reality the energy landscape is 3N-dimensional ( $\mathrm{N}$ : particle number). The common reaction coordinate contains the lithium hop as well as the back-and-forth dynamics of the oxygen. Of course, for different saddles and different oxygen atoms the specific energy landscape may somewhat look different. At $t_{\mathrm{S}}=0$ one may define an effective oxygen potential $V_{i}\left(R_{\mathrm{O}}\right)$. The index $i$ counts the different effective potentials, experienced by the different oxygen atoms. Now two length scales can be determined. $\xi_{\text {hom }}$ denotes the homogeneous length scale describing the range of possible fluctuations in the potential $V_{i}\left(R_{\mathrm{O}}\right)$. Close to the minima one expects in harmonic approximation $\xi_{\text {hom }} \propto$ $T^{1 / 2}$. In contrast, $\xi_{\text {het }}$ accounts for the heterogeneity when comparing hopping processes at different locations or different oxygen atoms. More specifically, it denotes the range of positions for which the different potentials $V_{i}\left(R_{\mathrm{O}}\right)$ display their respective minima. The two extreme limits $\xi_{\text {het }} \gg \xi_{\text {hom }}$ and vice versa are sketched in fig. 9. To elucidate the ratio of both length scales in the present case we would like to repeat two important observations, presented above. First, for oxygen atoms close to the saddle the position at $t_{\mathrm{S}}=0$ does not depend on its position 1 ps before the hop as derived from the comparison of the behavior of two different subensembles. Second, the oxygen distribution at $t_{\mathrm{S}}=0$ only shows an 
extremely weak temperature dependence. Both observations are compatible with the limit $\xi_{\text {het }} \gg \xi_{\text {hom }}$. In this case the position of any oxygen at $t_{\mathrm{S}}=0$ is very well characterized by its effective potential $V_{i}\left(R_{\mathrm{O}}\right)$ and does (in the extreme limit $\xi_{\text {hom }} \rightarrow 0$ ) not depend on its previous positions. Furthermore, since the effective energy landscape is to a large extent temperature independent one would not expect a major temperature dependence of the oxygen distribution, in agreement with observation. In any event, one may estimate $\xi_{\text {hom }} \ll 0.5 \AA$, i.e. the width of $\mathrm{P}_{\mathrm{NBO}}\left(r_{\mathrm{S}}, t_{\mathrm{S}}=0\right)$.

These results also reveal possible mechanisms for the dramatic slowing down of the ion dynamics upon immobilization of the network [7]. From the fig. [6] one can see that many oxygen atoms have to move as much as $0.5 \AA$ to arrive at their optimum position at $t_{\mathrm{S}}=0$. In case of an immobilized network this motion is not possible. This means that many oxygen atoms may be much further away from their optimum position during the transition as compared to $\xi_{\text {hom }}$. This automatically implies that the effective barrier for the combined lithium-oxygen transition will be much higher. This effect is sketched in fig. 8 where the suppression of the oxygen dynamics will lead to a significantly higher barrier of the transition in probably most of the hopping events. Of course, if the oxygen distance to a saddle is less than $1.5 \AA$ the resulting saddle is so high that effectively no lithium hopping is possible along this path. One can estimate from the oxygen distribution in fig. 5 at $t_{\mathrm{S}}=-2$ ps that this is the case for roughly $10 \%$ of all saddles. In any event, based on these considerations the dramatic increase of the activation energy upon immobilization can be understood.

As a gedankenexperiment one may wonder for which (unphysical) oxygen mass one has the transition between the mobile and the immobile limit. Based on the above interpretation of the correlated hopping motion it is required that the oxygen can follow the lithium ion during the time of a hop, which takes of the order of 500 fs. Thus the velocity of the oxygen atom should be decorrelated on time scales faster than 500 fs. For this purpose we analyze the quantity $w(t)$, which is the time derivative of the mean square displacement and twice the time integral of the velocity autocorrelation function [3, 27]. It is plotted in fig. [10. The short-time peak of $w(t)$ is a measure for the typical velocity decorrelation time. It is of the order of 30 fs for oxygen and thus much faster than the typical jump times of the lithium ions. Thus one can estimate that by increasing the oxygen mass by $(500 / 30)^{2}$ the slowing down of the lithium dynamics should start.

Actually, a similar estimation has been performed by Sunyer et al. for MD simulations of 
a sodium silicate system [5]. They explicitly observed the slowing down in dependence of the (artificial) mass of the network atoms. To rationalize the slowing down they have compared the fourier transform of the velocity correlation function of the sodium atoms with that of the oxygen atoms. This quantity expresses the vibronic density of states. They found that an overlap is important for the sodium dynamics. As soon as the oxygen atoms become too slow and the overlap decreases the sodium diffusion constant starts to decrease, too. This happens if the oxygen mass is scaled by a factor of the order of 100-1000. This estimation is close to the present case.

A further conclusion about the interrelation of lithium and network dynamics, as elucidated in this work, can be drawn from fig. 10, First, it is important to realize that around $t \approx 1 \mathrm{ps}$ there is a transition from localized lithium dynamics in a single site to hopping dynamics between adjacent sites. This has been explicitly shown in [3]. This time scale is also consistent with the present results because the hopping transition also roughly takes 1 ps. One can directly observe that up to 1 ps the observable $w(t)$ of the network atoms only weakly depends on temperature. This is in agreement with the expectation for the vibrational short-time limit $\left(w(t) \propto T^{1 / 2}\right)$. Around 1 ps the temperature dependence dramatically increases and is comparable with the temperature dependence of $w(t)$ for the lithium ions. Since in this time regime the lithium ions start to hop this correlation may reflect the sliding door mechanism as well as the network relaxation at ion sites, discussed in this work. For these mechanism one indeed expects a strong relation between network and lithium dynamics.

We just note in passing that the comparison of $w(t)$ for different species also has implications beyond the immediate topic of this work. In conductivity experiments one observes the sum of the transport of all charged constituents of the probe. Their contribution to $\sigma(\omega)$ is proportional to $w\left(t \approx \pi^{2} / \omega\right)[3,27]$. Thus one can conclude that for $t>1 \mathrm{ps}$, i.e. in the hopping regime, the contribution is mainly due to lithium dynamics.

In summary, we have elucidated in great detail the interrelation of lithium and network dynamics. It turns out that the reaction path for transition involves a significant oxygen dynamics. It can be characterized with a sliding door mechanism. There are strong indications that this dynamics is crucial to reduce the barrier height as compared to the case where the oxygen dynamics is suppressed due to network immobilization. 


\section{Acknowledgement}

Financial support from the DFG in the framework of SFB 458 is gratefully acknowledged. We appreciate helpful discussions with R. Banhatti, C. Cramer-Kellers, K. Funke, M.D. Ingram, H. Lammert, M. Vogel and D. Wilmer about this topic. 
[1] F. Howell, R. Bose, P. Macedo, and C. Moynihan, J. Phys. Chem., 1974, 78, 639.

[2] C. Angell, Solid State Ionics, 1986, 18-19, 72.

[3] A. Heuer, M. Kunow, M. Vogel, and R. Banhatti, Phys. Chem. Chem. Phys., 2002, 4, 3185.

[4] C. Angell, L. Boehm, P. Cheeseman, and S. Tamaddon, Solid State Ionics, 1981, 5, 659.

[5] E. Sunyer, P. Jund, and R. Jullien, J. Phys.: Condens. Matter, 2003, 15, L431.

[6] J. Habasaki, K. Ngai, and Y. Hiwatari, J. Chem. Phys., 2004, 120, 8195.

[7] A. Heuer, H. Lammert, and M. Kunow, Z. f. Phys. Chemie, 2004, 218, 1429.

[8] O. Anderson and D. Stuart, J. Am. Ceram. Soc., 1954, 37, 537.

[9] S. Martin and C. Angell, Solid State Ionics, 1986, 23, 185.

[10] P. Bandaranayake, C. Imrie, and M. Ingram, Phys. Chem. Chem. Phys., 2002, 4, 3209.

[11] M. Ingram, R. Banhatti, and I. Konidakis, Z. Phys. Chem, 2004, 218, 1401.

[12] J. Habasaki, K. Ngai, and Y. Hiwatari, J. Chem. Phys., 2004, 121, 925.

[13] H. Lammert, M. Kunow, and A. Heuer, Phys. Rev. Lett., 2003, 90, 215901.

[14] M. Vogel, Phys. Rev. B, 2004, 70, 139902.

[15] J. Horbach, W. Kob, and K. Binder, Phys. Rev. Lett., 2002, 88, 125502.

[16] A. Cormack, J. Du, and T. Zeitler, Phys. Chem. Chem. Phys., 2002, 4, 3193.

[17] J. Habasaki, I. Okada, and Y. Hiwatari, Phys. Rev. E, 1995, 52, 2681.

[18] J. Habasaki, I. Okada, and Y. Hiwatari, J. Non-Cryst. Solids, 1996, 208, 181.

[19] J. Habasaki, I. Okada, and Y. Hiwatari, Phys. Rev. B, 1997, 55, 6309.

[20] J. Habasaki and Y. Hiwatari, Phys. Rev. E, 1999, 59, 6962.

[21] R. Banhatti and A. Heuer, Phys. Chem. Chem. Phys., 2001, 3, 5104.

[22] J. Habasaki, Mol. Phys., 1990, 70, 513.

[23] J. Habasaki and I. Okada, Mol. Simul., 1992, 9, 319.

[24] J. Habasaki, I. Okada, and Y. Hiwatari, Mol. Simul., 1993, 10, 19.

[25] K. Refson, Comput. Phys. Commun., 2000, 126, 310.

[26] H. Doweidar, J. Non-Cryst. Solids, 1996, 194, 155.

[27] K. Funke, R. Banhatti, S. Brckner, C. Cramer, C. Krieger, A. Mandanici, C. Martiny, and I. Ross, Phys. Chem. Chem. Phys., 2002, 4, 3155. 
Fig. 1 Definition of the coordinate frame used in this work. The thin slice around $r_{s l}$ will be used further below.

Fig. 2 Temporal and spatial evolution of the relative density $\mathrm{P}_{\mathrm{HLi}}\left(r_{\mathrm{S}}, \theta_{\mathrm{S}}, t_{\mathrm{S}}\right)$ of hopping lithium.

Fig. 3 Pair correlation function $g_{\mathrm{Li}}(r)$ for lithium and non-bridging oxygen $(i=\mathrm{NBO})$.

Fig. 4 Temporal and spatial evolution evolution of the relative density $\mathrm{P}_{\mathrm{NBO}}\left(r_{\mathrm{S}}, \theta_{\mathrm{S}}, t_{\mathrm{S}}\right)$ of non-bridging oxygen adjacent to hopping lithium.

Fig. 5] Temporal and spatial evolution of the relative density $\mathrm{P}_{\mathrm{NBO}}\left(r_{\mathrm{S}}, t_{\mathrm{S}}\right)$ of nonbridging oxygen in a $0.5 \AA$ thin slice around the plane $\theta_{\mathrm{S}}=\pi / 2$.

Fig. 6 Relative density $\mathrm{P}_{\mathrm{NBO}}\left(r_{\mathrm{S}}, t_{\mathrm{S}}\right)$ of non-bridging oxygen in a $0.5 \AA$ thin slice around the plane $\theta_{\mathrm{S}}=\pi / 2$ for different subensembles $A_{1}$ and $A_{2}$ compared to $\mathrm{P}_{\mathrm{NBO}}\left(r_{\mathrm{S}}, t_{\mathrm{S}}\right)$ for all non-bridging oxygens in this slice. Particles belonging to subensemble $A_{1}$ are located at $r_{\mathrm{S}} \leq 1.5 \AA$ at $t_{\mathrm{S}}=-1 \mathrm{ps}$. Particles belonging to subensemble $A_{2}$ are located at $r_{\mathrm{S}} \leq 1.5 \AA$ at $t_{\mathrm{S}}=-2 \mathrm{ps}$.

Fig. 7 Temporal and spatial evolution of the relative density $\mathrm{P}_{\mathrm{NBO}}\left(r_{\mathrm{I}}, t_{\mathrm{S}}\right)$ of nonbridging oxygen in a hemisphere around $\mathbf{r}_{\text {init }}$ covering angles $\pi / 2 \leq \theta_{\mathrm{I}} \leq \pi$.

Fig. 8 Schematic 2D energy landscape, reflecting the cooperative motion of the lithium and an oxygen atom. The thick line describes the reaction coordinate. The straight line for constant $r_{O}$ describes the transition path without oxygen dynamics. In contrast, along the straight line for constant $r_{\mathrm{Li}}$ one can read off the effective potential $V_{i}\left(R_{\mathrm{O}}\right)$ for the oxygen atom at $t_{\mathrm{S}}=0$.

Fig. 9 Sketch of the two extreme scenario of the effective potentials $V_{i}\left(R_{\mathrm{O}}\right)$, experienced by the oxygen atoms at time $t_{\mathrm{S}}=0$. (a) Limit of strong curvature; (b) Limit of weak curvature.

Fig. 10 Time derivative $w(t)$ of the mean square displacement for all particle species in the system at three different temperatures. 


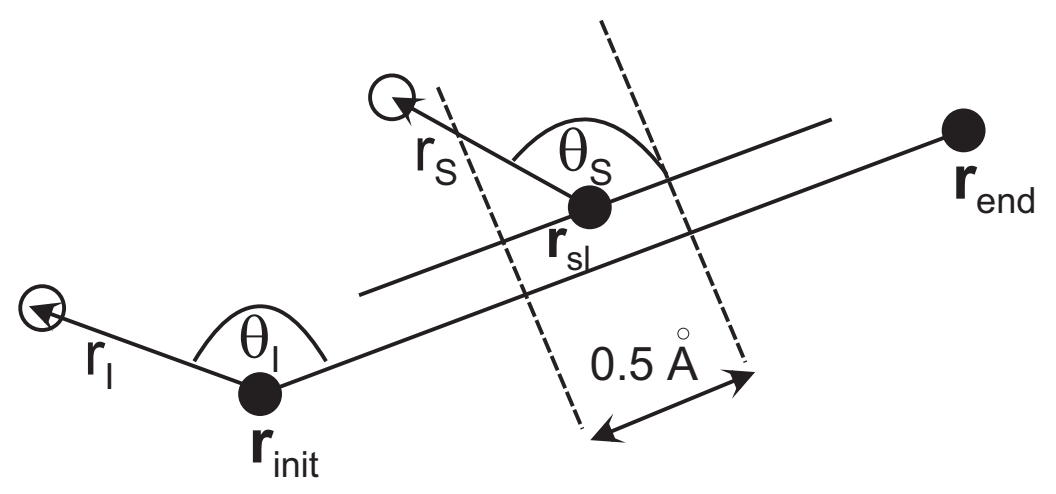

FIG. 1: 

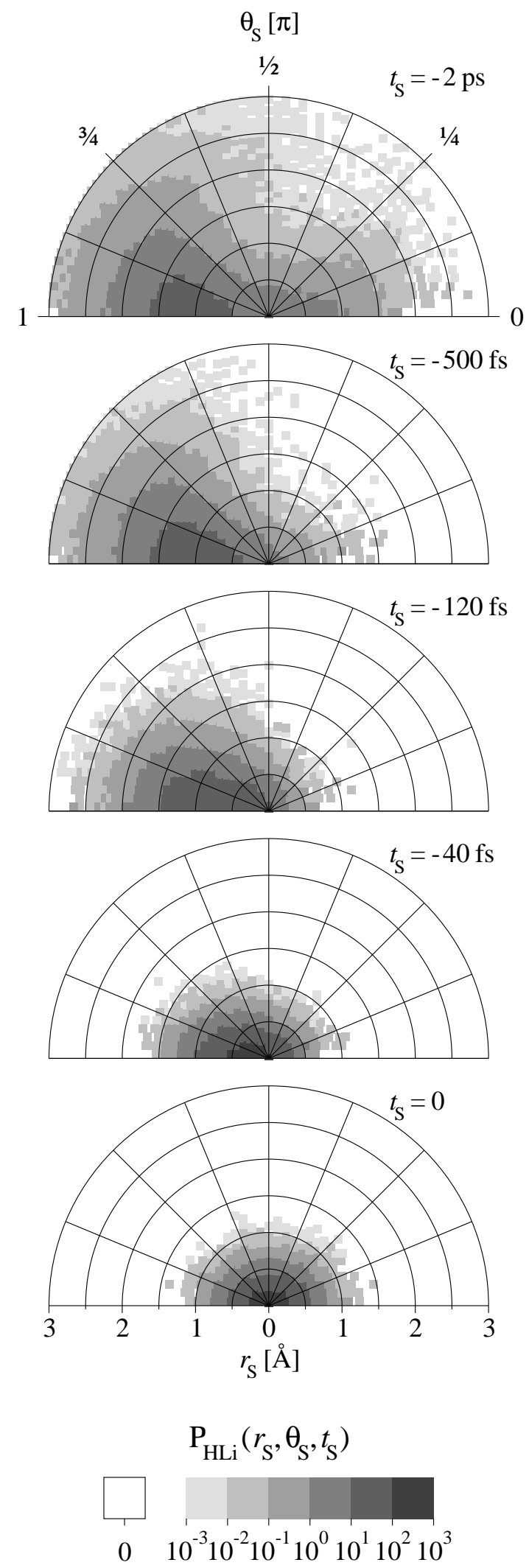

FIG. 2: 


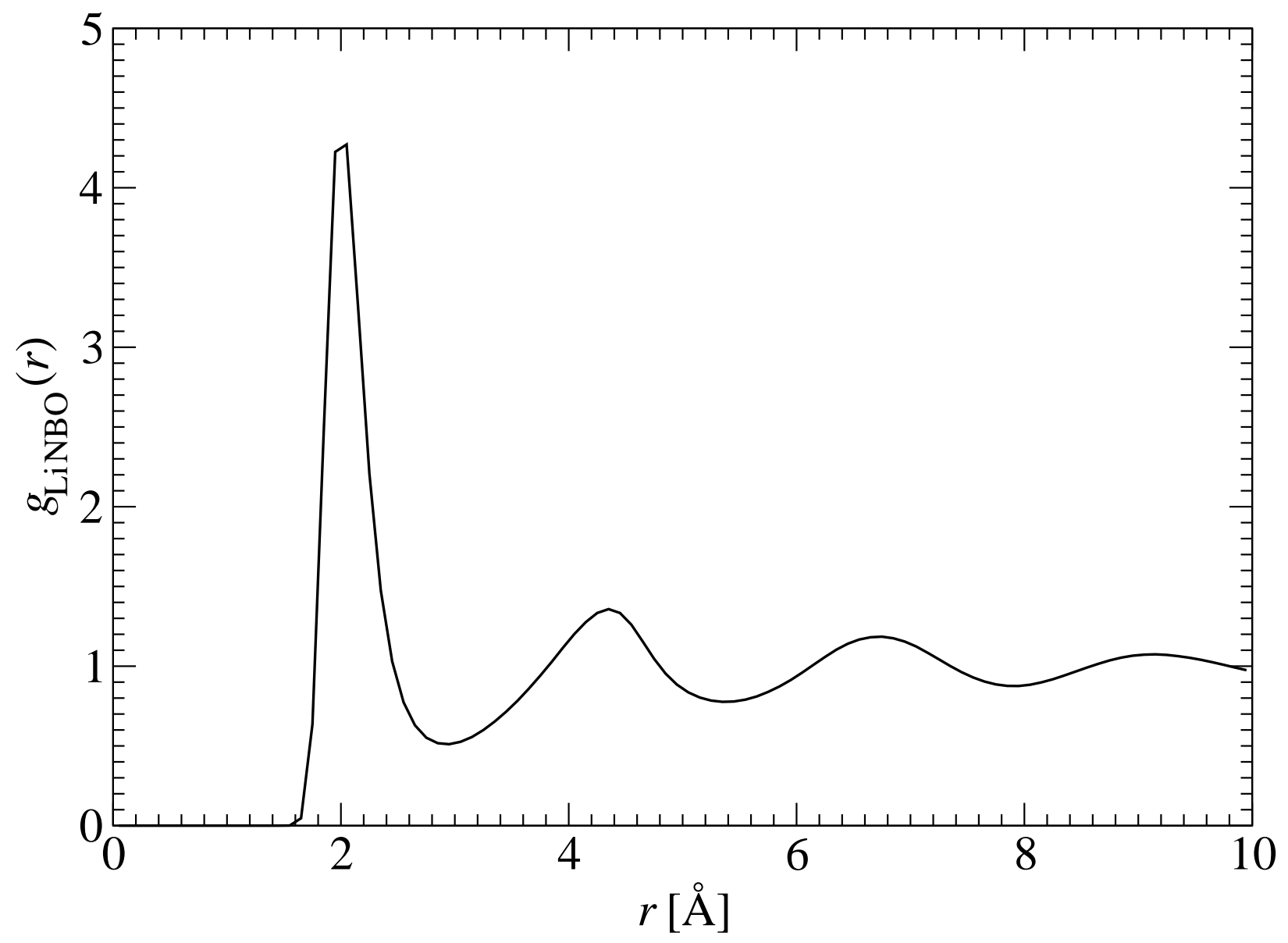

FIG. 3: 


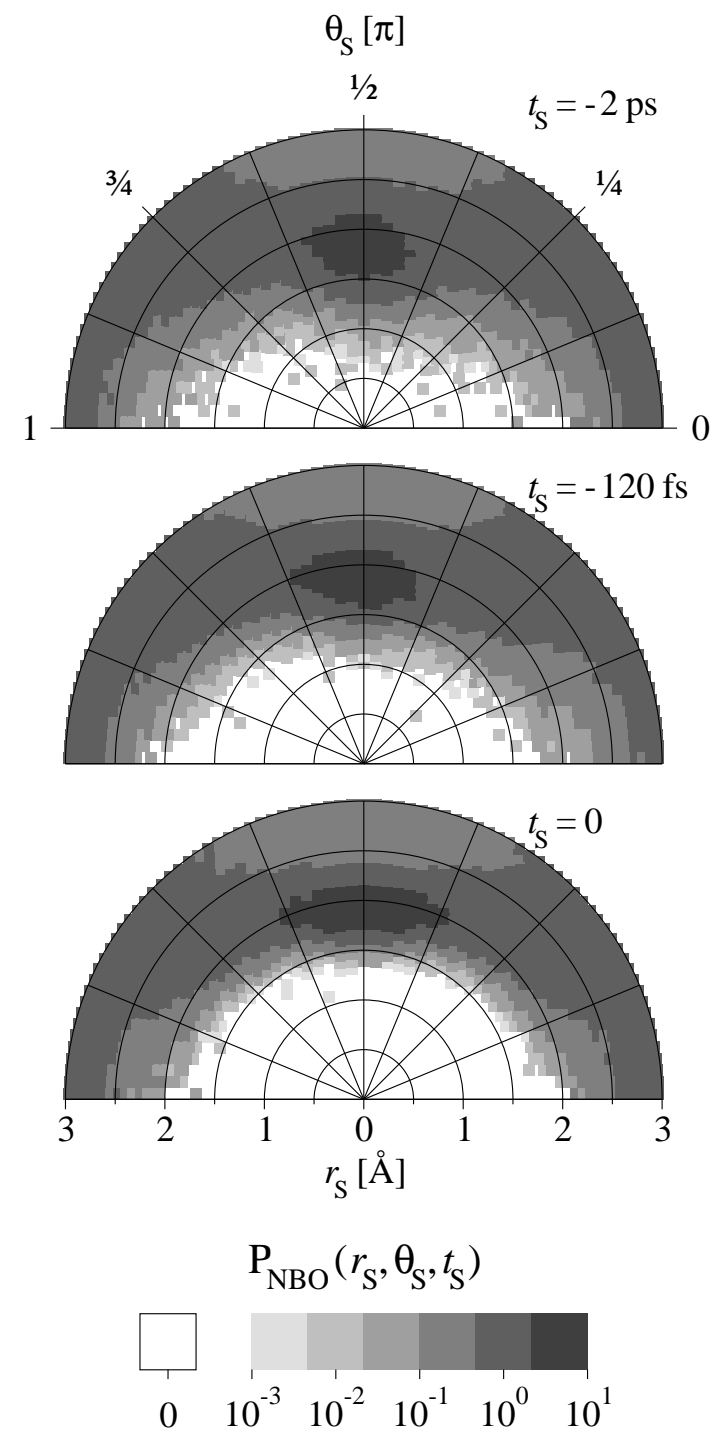

FIG. 4: 


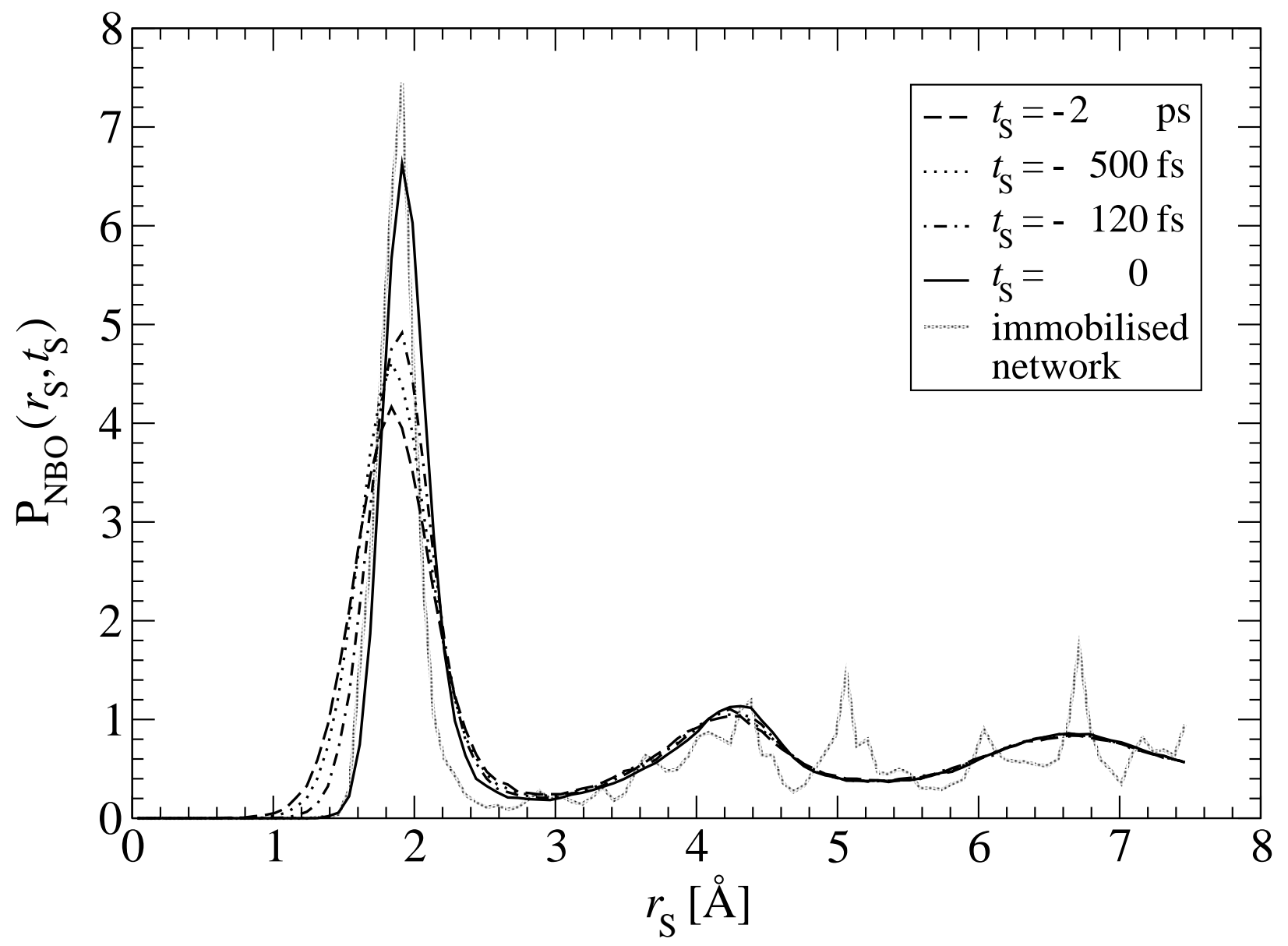

FIG. 5: 


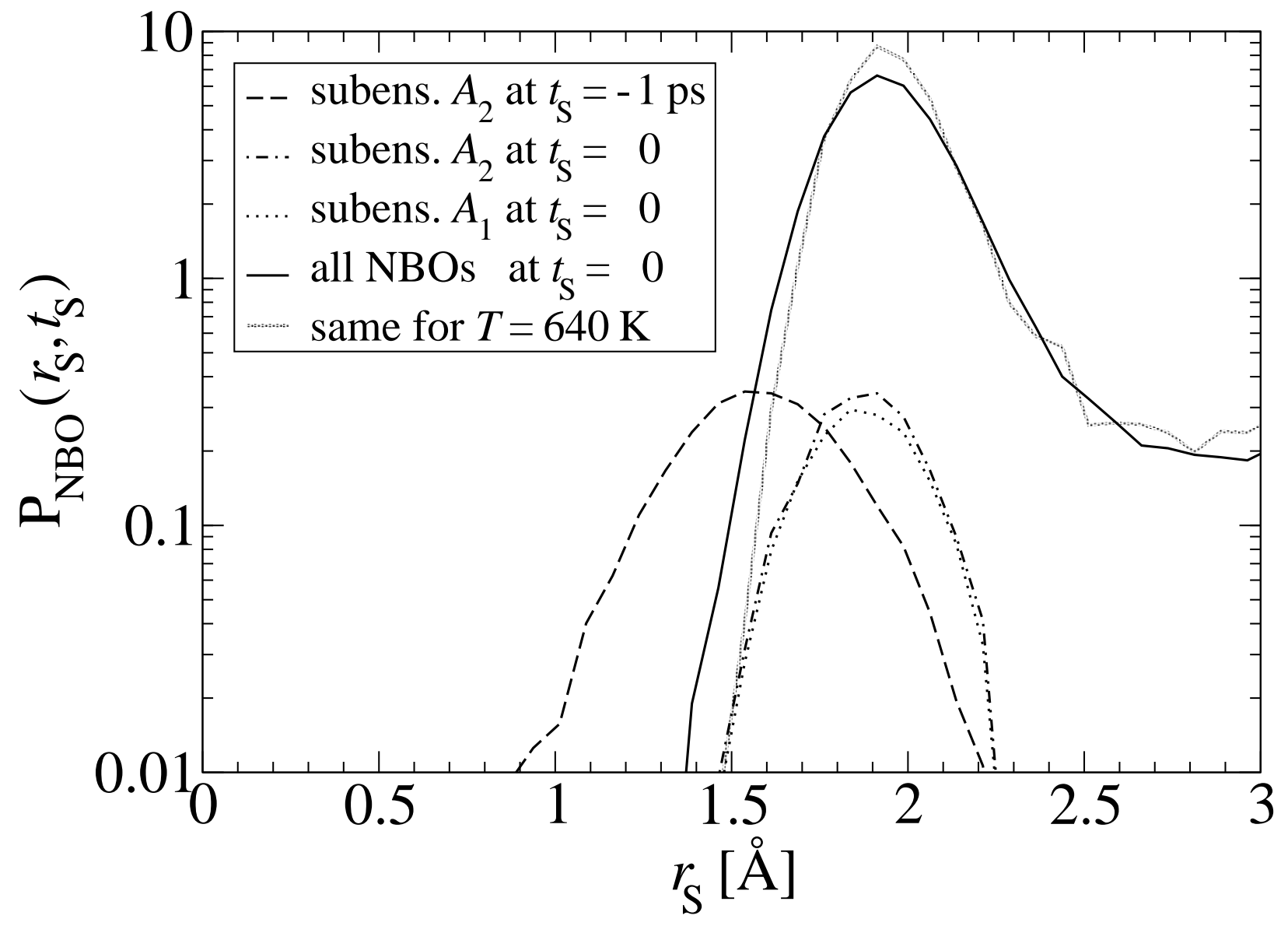

FIG. 6: 


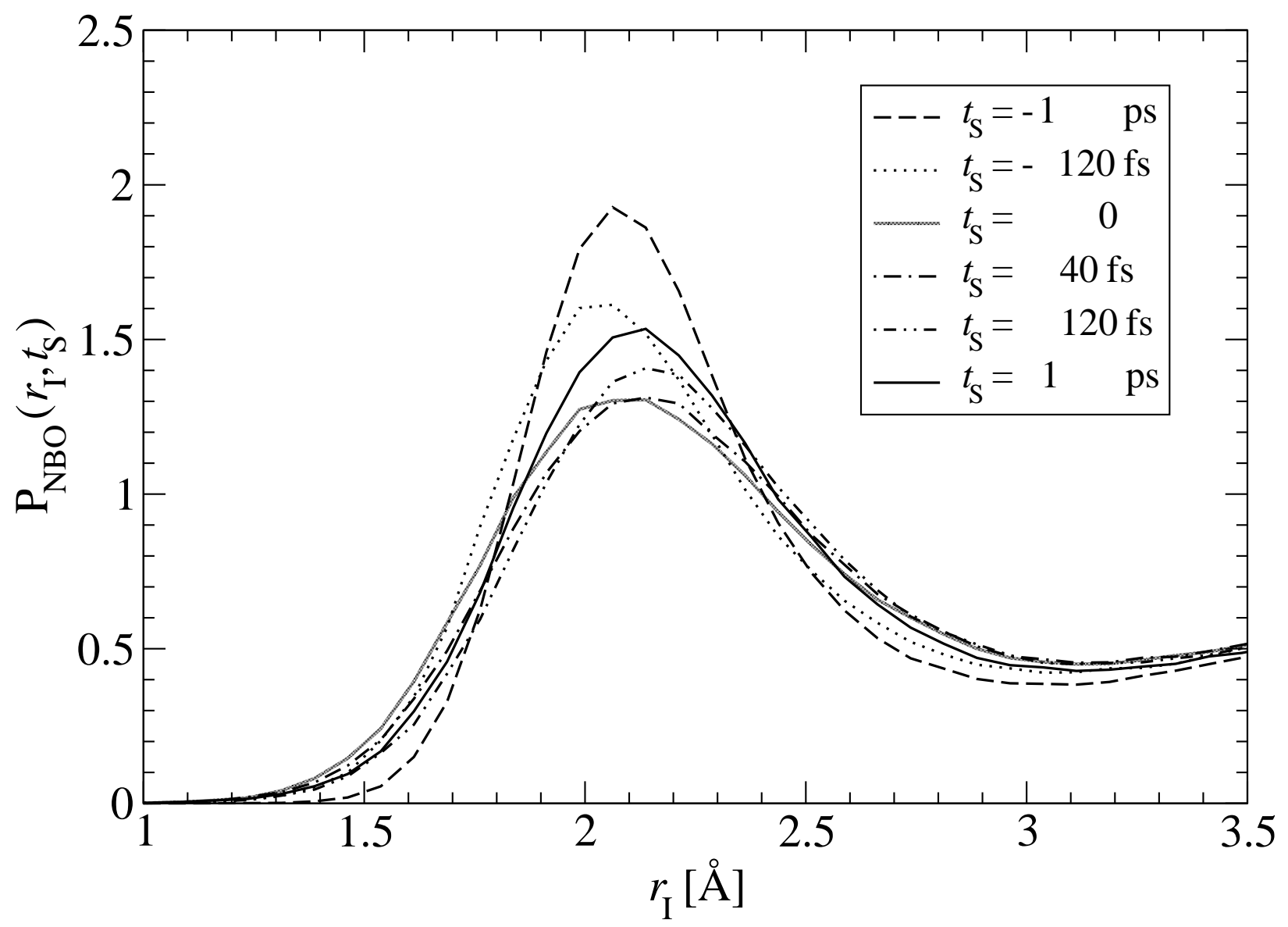

FIG. 7: 


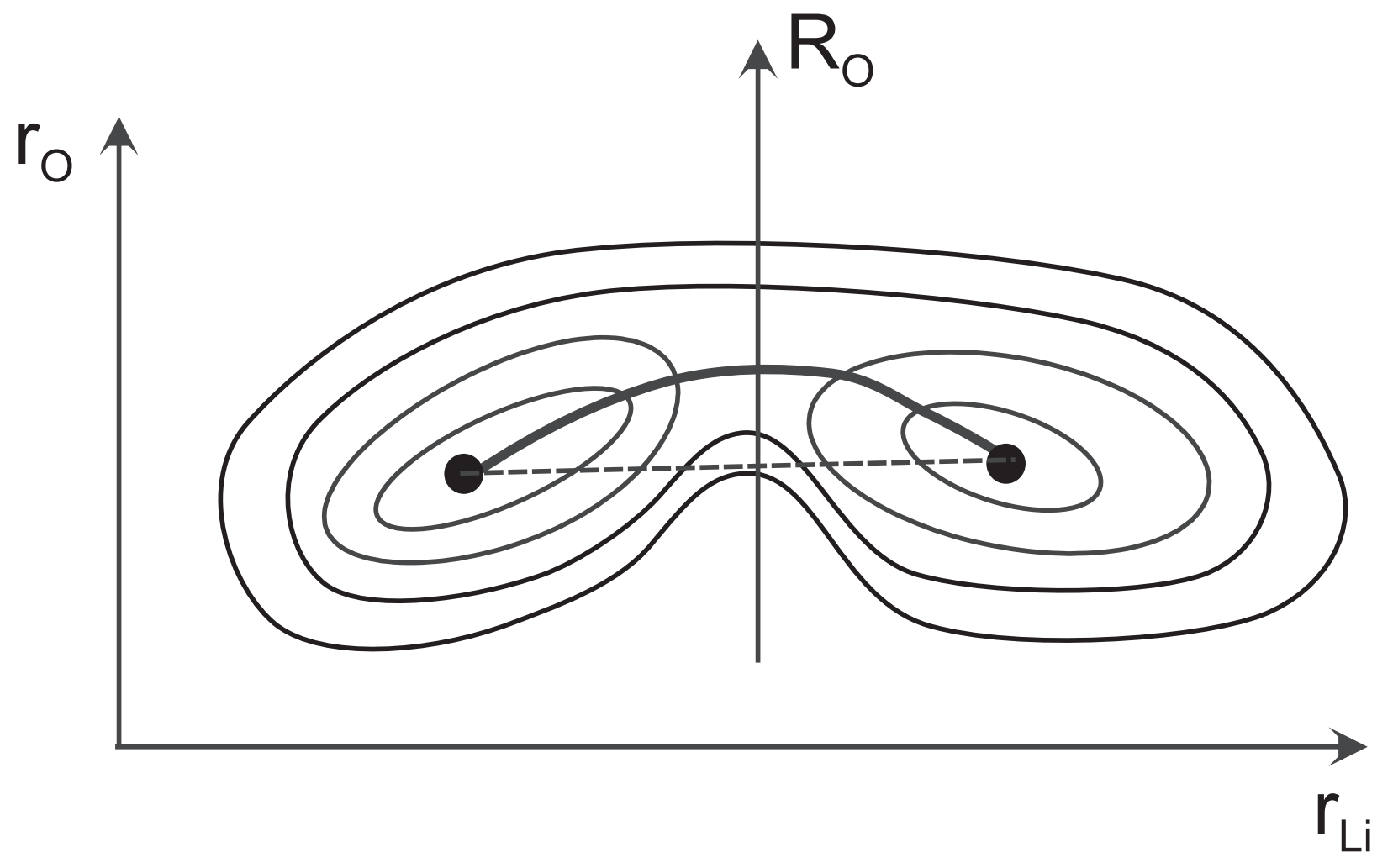

FIG. 8:

(a)

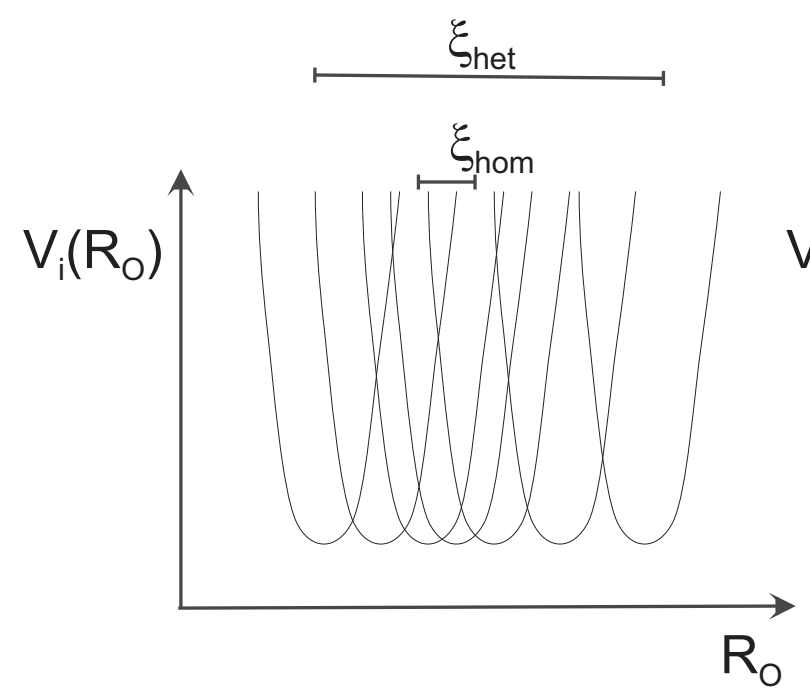

(b)

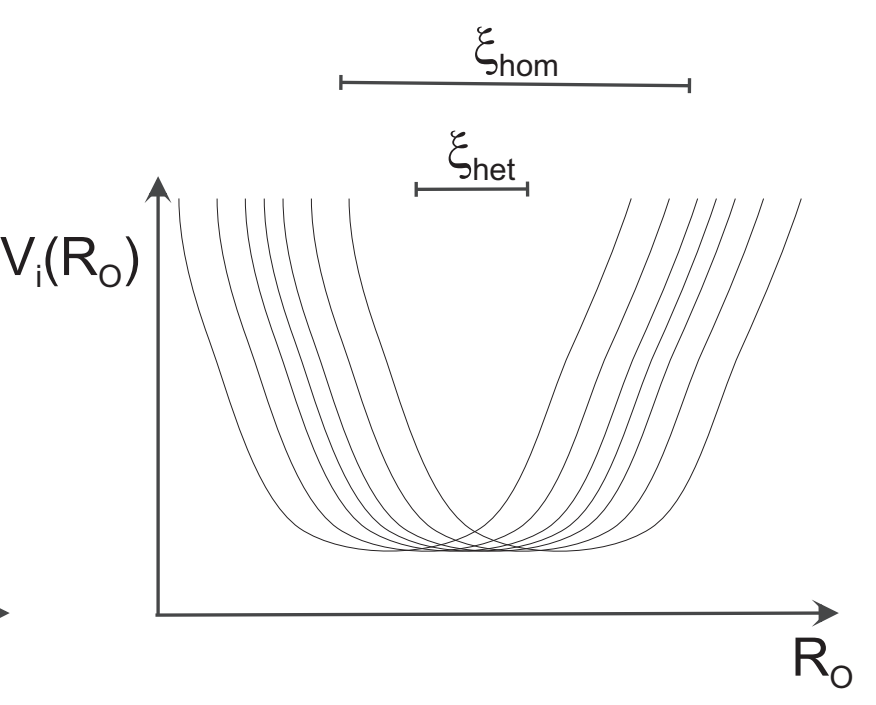

FIG. 9: 


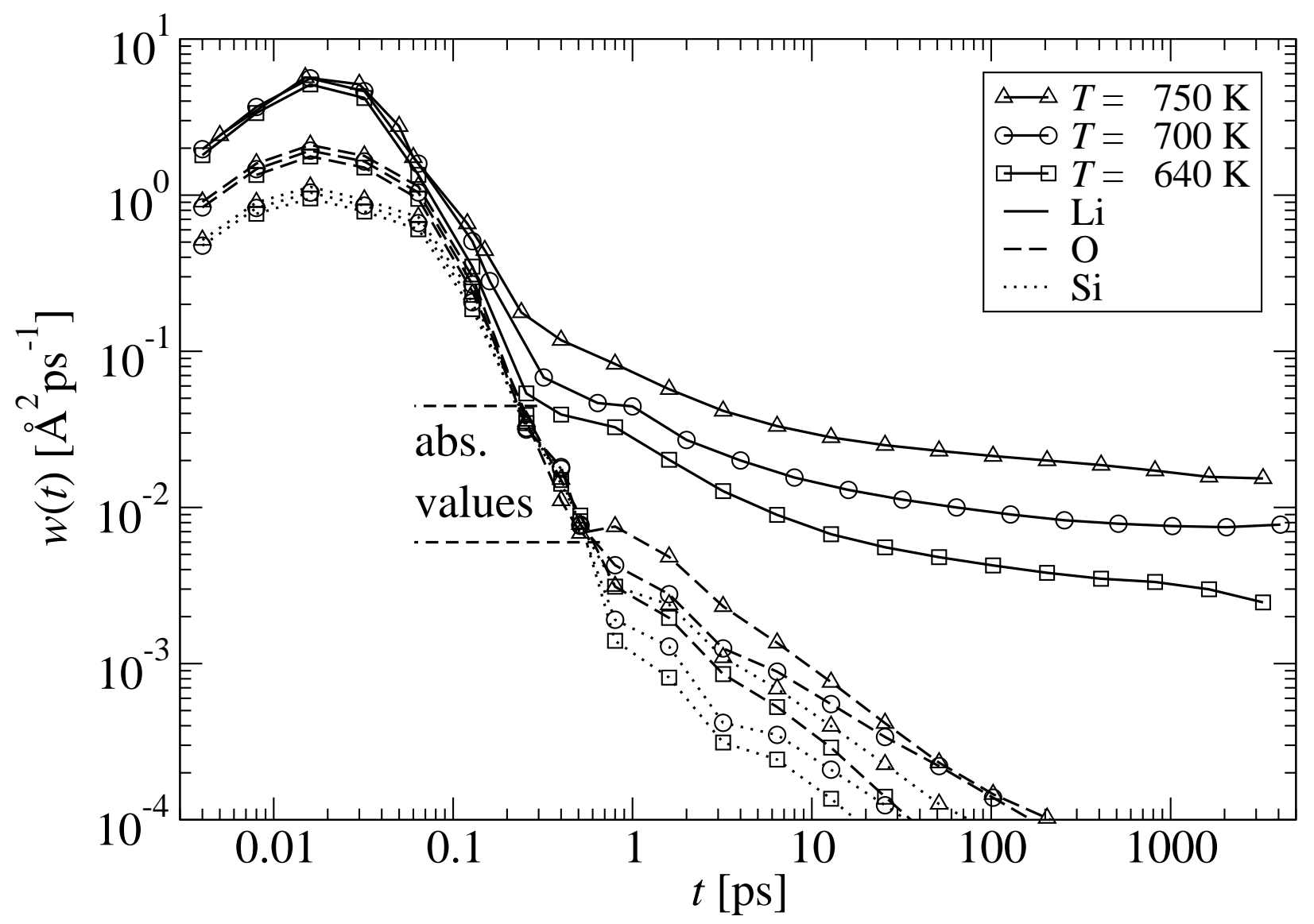

FIG. 10: 